\title{
Fiber-Optic pH Sensor with Monolayer Indicator
}

\author{
Yuji Kawabata, Kiyoshi Tsuchida, Totaro Imasaka and Nobuhiko IshibashI \\ Department of Applied Analytical Chemistry, Faculty of Engineering, Kyushu University, \\ Hakozaki, Fukuoka 812
}

\begin{abstract}
A fiber-optic $\mathrm{pH}$ sensor has been constructed by immobilizing fluorescein isothiocyanate (FITC) on the end surface of the optical fiber. The $\mathrm{pH}$ response is reversible in the range $4.0-6.9$, precision being \pm 0.1 . Due to implantation of FITC as a monolayer, the intrinsic response time is less than $10 \mathrm{~ms}$.
\end{abstract}

Keywords Optrode, pH sensor, optical fiber, fluorescein, monolayer

A fiber-optic chemical sensor (optrode) is an attractive detector for bioanalysis in physiological substances. Since the signal is optically detected, the sensor is not interfered by electrical noise. High transmittance of the light in an optical fiber offers a new analytical technique for remote sensing. Various fiber-optic sensors based on fluorimetry have been developed. These sensors are useful for the measurement of $\mathrm{pH}^{1}, \mathrm{O}_{2}{ }^{2}$, and metal ions. ${ }^{3}$ In these studies chemical reagents are immobilized on the polymer support which is attached on a common end surface of a bifurcated fiber-optic bundle. This type of sensor gives a large fluorescence signal, since a sufficient amount of the fluorescence reagent is bound on the support by adsorption or ion exchange. However, it sometimes suffers from its slow response time and from poor mechanical stability. Recently, Munkholm et al. have proposed the fiber-optic $\mathrm{pH}$ sensor in which an indicator phase is polymerized on an end surface of a single optical fiber. ${ }^{4}$ Their sensor have a response time of $9 \mathrm{~s}$ or less, but it shows the lack of sensor reproducibility due to difficulty in controlling the polymerization process. The response time and sensor reproducibility are further improved by decreasing the thickness of the reagent to a monolayer. ${ }^{5,6}$

In this study we develop a new type of $\mathrm{pH}$ sensor. The indicator is covalently immobilized as a monolayer on the end surface of the optical fiber, and transmission of exciting laser beam and collection of fluorescence are achieved through a single optical fiber. We investigate the response time of the sensor and the $\mathrm{pH}$ range useful for analytical measurements.

\section{Experimental}

Apparatus

The block diagram of the experimental apparatus is shown in Figure 1. An argon ion laser (NEC,
GLG3200, $488 \mathrm{~nm}, 2 \mathrm{~mW}$ ) is used as an exciting source. The laser beam is modulated to square waves at $53 \mathrm{~Hz}$ by a chopper driven by an induction motor (Oriental Motor, 2IK6RA-A) and is split by a quartz plate. The reflected beam is detected by a photodiode (Hamamatsu Photonics, S1190) and the output signal is fed into a lock-in amplifier (NF Circuit Design Block, LI-570) as a reference signal. The transmitted beam passes through a hole (diameter $3 \mathrm{~mm}$ ) located at the center of mirror 2 and is focused onto the front surface of a quartz optical fiber (Fujikura, G800/1100, core diameter $800 \mu \mathrm{m}$, cladding diameter $1100 \mu \mathrm{m}$ ). A $\mathrm{pH}$ indicator of fluorescein isothiocyanate (FITC) is immobilized onto the end surface of the optical fiber and is irradiated by the laser beam introduced into the fiber. Fluorescence from immobilized FITC is collected by the same optical fiber and is collimated by lens 1 . It is reflected by mirror 2 and is focused by lens 2 onto the entrance slit of a monochromator (Jasco,

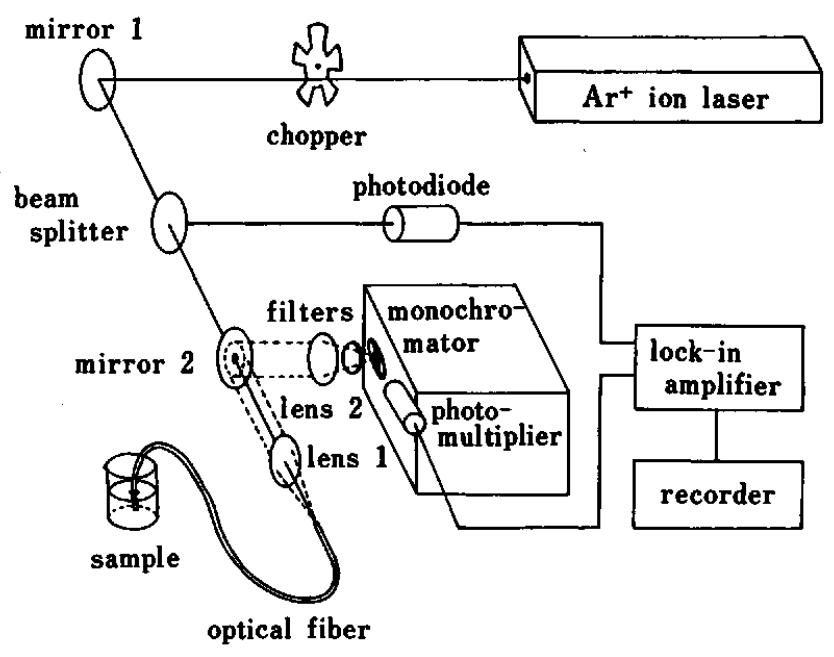

Fig. I Schematic diagram of fiber-optic pH sensor. 
CT-50, $520 \mathrm{~nm}$ ) equipped with a photomultiplier tube (Hamamatsu Photonics, R212) after passing through a cut-off filter (Fuji Film, SC-50, cut-off wavelength $500 \mathrm{~nm}$ ) and a band pass filter (Fuji Film, BPB-53, $530 \mathrm{~nm}$, bandwidth $60 \mathrm{~nm}$ ). The output signal is fed through the lock-in amplifier to a chart recorder (Rikadenki, R-50).

The intrinsic response time of the sensor is measured for FITC immobilized on a glass plate for microscopy (Matsunami Glass Ind.) in the same manner described for the optical fiber. The glass plate is soaked in a buffer solution at $\mathrm{pH} 4.0$ and is wiped to remove the excess solution on the glass plate. A buffer solution at pH 9.2 is dropped onto the surface of the glass plate to change $\mathrm{pH}$ immediately. Fluorescence from immobilized FITC is detected by the photomultiplier tube through the monochromator. The transient signal is amplified a hundred times by an amplifier (NF Circuit Design Block, LI-75A) and is measured by a digitizer (Autnics, S-210). The waveform in the memory is recorded by the chart recorder.

A pH meter (TOA Electronics Ltd., HM-5B) is used to measure $\mathrm{pH}$ of a sample solution. The absorption spectrum of immobilized FITC is measured by a spectrophotometer (Shimadzu, UV-140-02).

\section{Reagents}

A pH indicator of FITC was purchased from Dojindo Laboratories and 3-aminopropyldimethylethoxysilane from Petrarch System Inc. Tosyl chloride was obtained from Wako Pure Chemical Industries and organic solvents such as toluene from Kishida Chemical Co. The buffer solutions at $\mathrm{pH} 4.0$ (phthalate buffer), $\mathrm{pH} 6.9$ (phosphate buffer), and $\mathrm{pH}$ 9.2 (borate buffer) were also purchased from Kishida Chemical Co. and the solutions at $\mathrm{pH} 5.1$ and 6.1 were prepared by addition of $0.1 \mathrm{M} \mathrm{NaOH}$ to $0.1 \mathrm{M} \mathrm{HCl}$.

\section{Immobilization procedure}

The $\mathrm{pH}$ indicator of FITC was immobilized as a monolayer on the end surface of the optical fiber by the following procedure. First, the end surface of the optical fiber was polished by fine emery paper and was washed with acetone. The surface was dipped into 20 $\mathrm{ml}$ of toluene containing $0.3 \mathrm{ml}$ of 3-aminopropyldimethylethoxysilane and $2 \mathrm{mg}$ of tosyl chloride at $75^{\circ} \mathrm{C}$ for $2 \mathrm{~h}$. The fiber surface was washed with ethanol and water in turn. By this procedure an amino group was implanted on the optical filber. Secondly, $20 \mathrm{ml}$ of the aqueous solution containing $2 \mathrm{mg}$ of FITC and $0.5 \mathrm{~g}$ of sodium acetate was prepared. The optical fiber was dipped into the solution at a room temperature for $12 \mathrm{~h}$ to combine FITC with the amino group. The modified surface of the optical fiber was washed with a copious amount of water.

\section{Results and Discussion}

At low pH, FITC in solution has an absorption band at $430 \mathrm{~nm}$ and is nonfluorescent. With increasing $\mathrm{pH}$ from 4.0 to 9.0 the absorption band appears at $490 \mathrm{~nm}$, which corresponds to absorption of the species fluorescing at $520 \mathrm{~nm}$. The fluorescence intensity is proportional to absorbance at $490 \mathrm{~nm}$. Therefore, the $\mathrm{pH}$ of the solution can be measured by monitoring the fluorescence intensity at $520 \mathrm{~nm}$.

The response curve of the constructed sensor is shown in Fig. 2. The pH response was reversible in the range 4.0-6.9, and the upper limit of the response range was 7.4. FITC was also immobilized on the glass plate and absorbance of immobilized FITC was measured. The value at $490 \mathrm{~nm}$ changed from $6 \times 10^{-4}$ at $\mathrm{pH} 4.0$ to $2 \times 10^{-3}$ at $\mathrm{pH} 7.4$, and it was constant in the range 7.4-9.2. Since absorbance of immobilized FITC is sufficiently low, signal saturation for the fiberoptic sensor is not due to the inner-filter effect by reabsorption of fluorescence. The proton dissociation constants were calculated from the change of the fluorescence intensity with $\mathrm{pH}$. They were $10^{-6.5}$ and $10^{-5.9}$ for free and immobilized FITC, respectively. The proton dissociation constant is often varied by immobilization of the reagent on the solid support.

The observed rise time of the sensor was $9 \mathrm{~s}$ for $63 \%$ (1-(1/e)) response, which was determined by a time constant of the lock-in amplifier. The long time constant was necessary to improve the signal-to-noise ratio since fluorescence from the monolayer was very weak. We used a laser as an exciting source for sensitive fluorescence detection, but decomposition of immobilized FITC from the optical fiber occurred by irradiation of the laser with an output power exceeding $30 \mathrm{~mW}$. Then, the signal-to-noise ratio could not be further improved by increasing the output power of the laser.

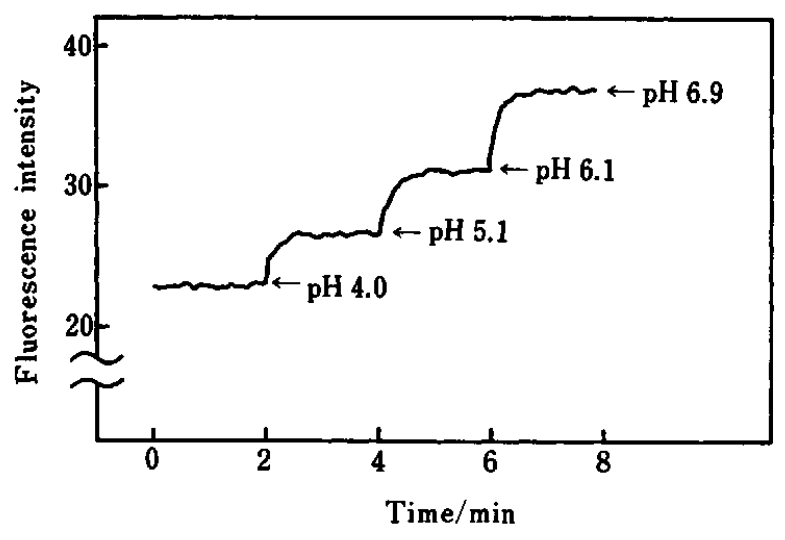

Fig. 2 Response curve of fiber-optic pH sensor. The $\mathrm{pH}$ was changed by immersing the sensor to the solution with different $\mathbf{p H}$ value. 


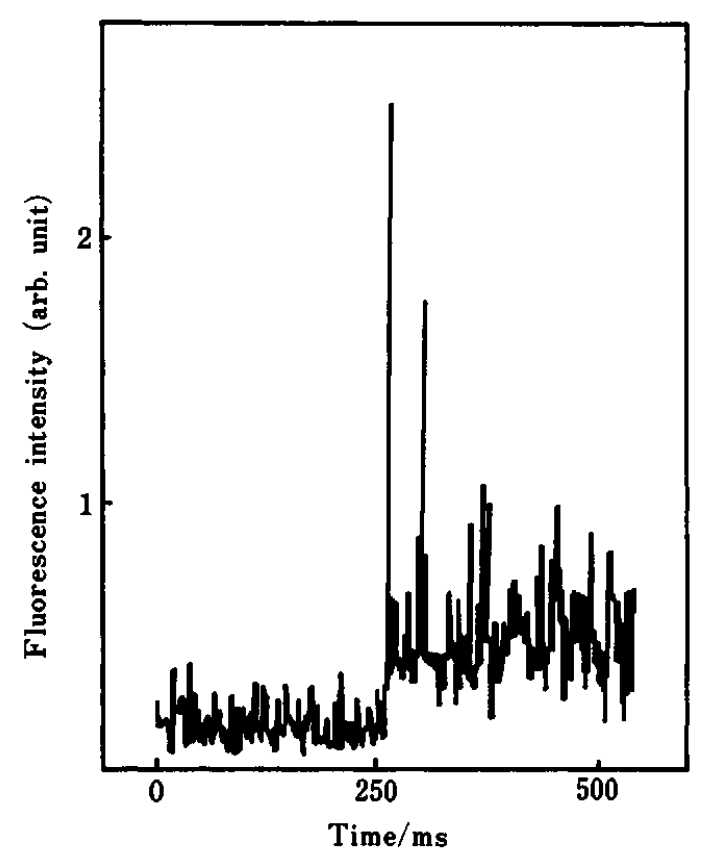

Fig. 3 Transient response curve of immobilized FITC. The response time is less than $10 \mathrm{~ms}$. The impulse signal immediately after $\mathrm{pH}$ change ( $\mathrm{pH} 4.0$ to 9.2 ) is originated by light scattering from the droplet. This impulse signal also appeared when the glass plate was used without immobilization of FITC.

A background signal was sufficiently reduced by using two filters, but it was still negligible. The intensity of the background signal gradually decreased with increasing the detection wavelength from the excitation line. The background may probably be due to scattering at the end surface of the optical fiber.

The sensitivity of the sensor was constant during at least 3 days. The error in the $\mathrm{pH}$ measurement was \pm 0.1 for the constructed sensor, which was determined by fluctuation of the background signal. Further improvement of the optical detection system and the use of the more photoresistive indicator may increase the signal-to-noise ratio, resulting a short time constant of the electronics.

The transient response of immobilized FITC on the glass plate is shown in Fig. 3. When the buffer solution was dropped onto the surface of the glass plate, the fluorescence intensity quickly increased by the $\mathrm{pH}$ change. It shows that the response time of the sensor was less than $10 \mathrm{~ms}$. Protonation and deprotonation might occur in the order of nanoseconds, and the response time is practically limited by the rate of the mass transfer process and the response time of the electronics. The potential of the glass electrode is reported to reach a constant value within $2 \mathrm{~s}$ by immersing in liquid solution with different $\mathrm{pH} .{ }^{8}$ Thus the fiber-optic $\mathrm{pH}$ sensor with a monolayer indicator essentially has a distinct advantage with respect to the response time over the conventional glass electrode.

This research was partly supported by a Grant-in-Aid for Scientific Research from the Ministry of Education of Japan and the Grant-in-Aid on Special Project Research for "Organic Thin Films for Information Conversion" from the Ministry of Education, Science and Culture.

\section{References}

1. L. A. Saari and W. R. Seitz, Anal. Chem., 54, 821 (1982).

2. J. I. Peterson, R. V. Fitzgerald and D. K. Buckhold, Anal. Chem., 56, 62 (1984).

3. Z. Zhujun and W. R. Seitz, Anal. Chim. Acta, 171, 251 (1985).

4. C. Munkholm, D. R. Walt, F. R. Milanovich and S. K. Klainer, Anal. Chem., 58, 1427 (1986).

5. E. Urbano, H. Offenbacher and O. S. Wolfbeis, Anal. Chem., 56, 427 (1984).

6. O. S. Wolfbeis, H. Offenbacher, H. Kroneis and $\mathbf{H}$. Marsoner, Mikrochim. Acta [Wien] 1984 I, 153.

7. Z. Zhujun and W. R. Seitz, Anal. Chim. Acta, 160, 47 (1984).

8. G. A. Perley, Anal. Chem., 21, 559 (1949).

(Received October 6, 1986) (Accepted November 10, 1986) 